case report

\title{
Obliterative hepatocavopathy - ultrasound and cavography findings
}

\author{
Ramazan Kutlu \\ Department of Radiology, Inonu University School of Medicine, Malatya, Turkey
}

\begin{abstract}
Backgound. Obliterative hepatocavopathy is a relatively new term for the definition of primary inferior vena cava thrombosis and membranous obstruction of inferior vena cava that were included in Budd-Chiari syndrome.
\end{abstract}

Case report. In this report, cavography and ultrasound findings of a case with a complete occlusion of inferior vena cava in a 36 year-old female patient who has been followed up with the diagnosis of hepatitis $B$ related cirrhosis for 5 years were presented.

Conclusions. Although this disease classically treated by surgery (e.g. portal or mesenteric-systemic shunting, liver transplantation), also interventional radiology procedures e.g. transjugular intrahepatic portosystemic shunting, percutaneous hepatic vein angioplasty and/or stent placement), now play an important role in the management ${ }^{4}$.

Key words: obliterative hepatocavopathy; cavography; ultrasound; inferior vena cava

\section{Introduction}

The complete inferior vena cava (IVC) obstruction develops as a result of primary IVC thrombosis. ${ }^{1}$ Although in the earlier literature this entity was included in BuddChiari syndrome, obliterative hepatocavopathy $(\mathrm{OH})$ is currently being used to define this condition. ${ }^{1}$ In this report, we present

Received 20 September 2008

Accepted 28 October 2008

Correspondence to: Assoc. Prof. Ramazan Kutlu, M.D., PhD, Department of Radiology, Inonu University School of Medicine, Turgut Ozal Medical Center, 44069 Malatia, Turkey. Phone: +90 4223410660 (Extn: 5703); Fax: +90 4223410834 (Attn: Dr. Ramazan Kutlu); E-mail: rkutlu@inonu.edu.tr cavography and ultrasound features of this rare entity in a 36 year-old female patient.

\section{Case report}

A 36 year-old female patient who has been followed with the diagnosis of chronic liver disease (Child B, score 7) due to hepatitis $B$ for 5 years was referred to our radiology department for portal Doppler evaluation. She had splenomegaly and moderate ascites. Upper gastrointestinal endoscopy revealed esophageal varices. She had been receiving beta blockers and diuretics. Her CA-125 level was found to be increased (234 U/mL). 

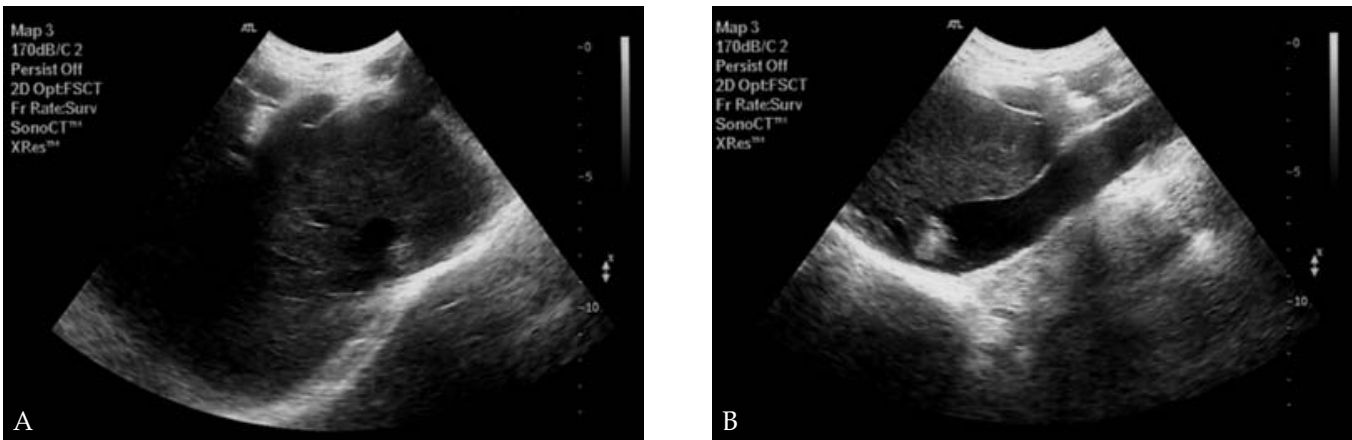

Figure 1. Gray scale axial (A) and sagittal (B) US images show hyperechoic, solid material at the posterolateral part of distal IVC.

Gray scale (Figure $1 \mathrm{~A}, \mathrm{~B})$ and Doppler (Figure 2 A-C) US examinations showed compressed hepatic veins, hepatopedal portal flow, and no blood flow into the right atrium through distal IVC which was thrombosed. The abdominal CT examination revealed the absence of intrahepatic portion of IVC. The patient didn't undergo
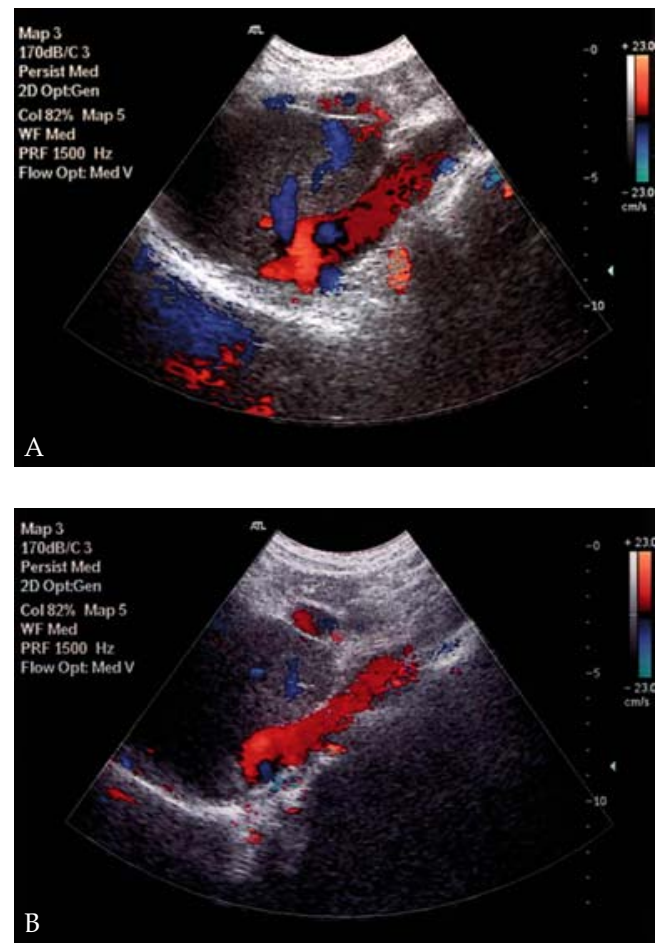

MR examination due to claustrophobia. Cavography was performed using right femoral vein access. Firstly, a large vein was erroneously accessed and a test injection before introducer placement demonstrated that this vein was a large collateral vein extending to the right side of the body and ultimately draining to the intercostal, pericardiophrenic and right subclavian vein (Figures 3 A-E). Secondly, the right femoral vein was catheterized and inferior cavography revealed a complete occlusion of distal $4 \mathrm{~cm}$ of IVC connecting to the right atrium and retrograde flow into the prominent collaterals in the late series (Figures $4 \mathrm{~A}, \mathrm{~B}$ ). The selective catheterization of the hepatic veins showed hepatic vein confluence and weak filling (Figure 5A) and a small vein below hepatic confluence draining to IVC (Figure 5B). Superior vena cavography

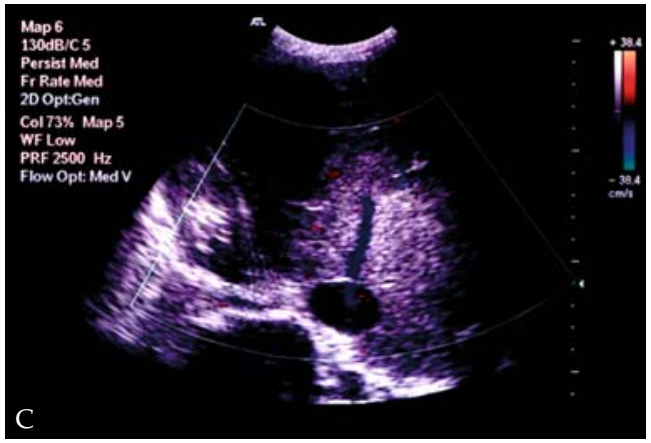

Figure 2. Sagittal (A, B) Doppler US images slight filling of hepatic vein and complete occlusion of distal IVC. Subcostal oblique image $(C)$ reveals a relatively small hepatic vein below the hepatic confluence. 

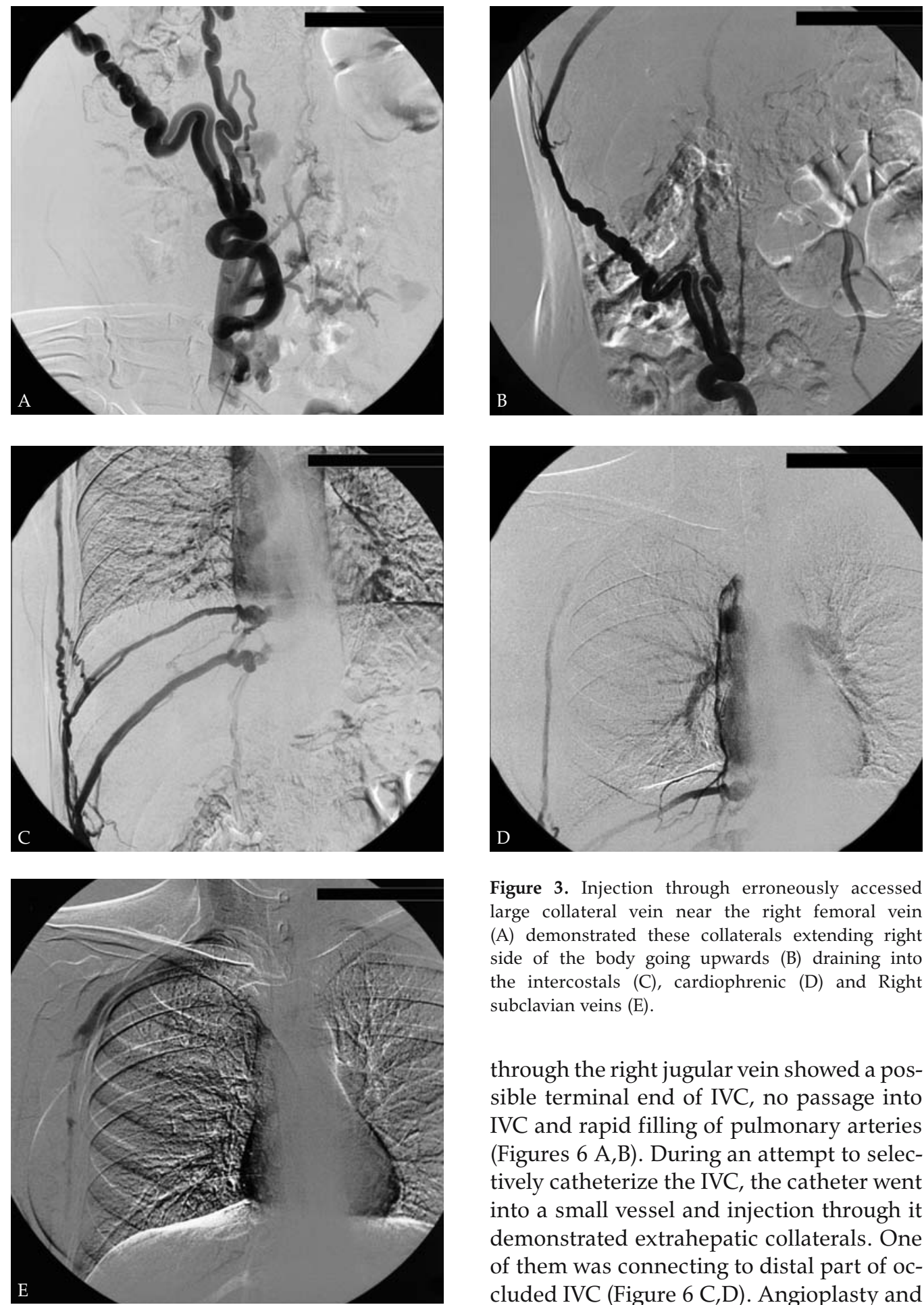

Figure 3. Injection through erroneously accessed large collateral vein near the right femoral vein (A) demonstrated these collaterals extending right side of the body going upwards (B) draining into the intercostals (C), cardiophrenic (D) and Right subclavian veins (E).

through the right jugular vein showed a possible terminal end of IVC, no passage into IVC and rapid filling of pulmonary arteries (Figures 6 A,B). During an attempt to selectively catheterize the IVC, the catheter went into a small vessel and injection through it demonstrated extrahepatic collaterals. One of them was connecting to distal part of occluded IVC (Figure 6 C,D). Angioplasty and 

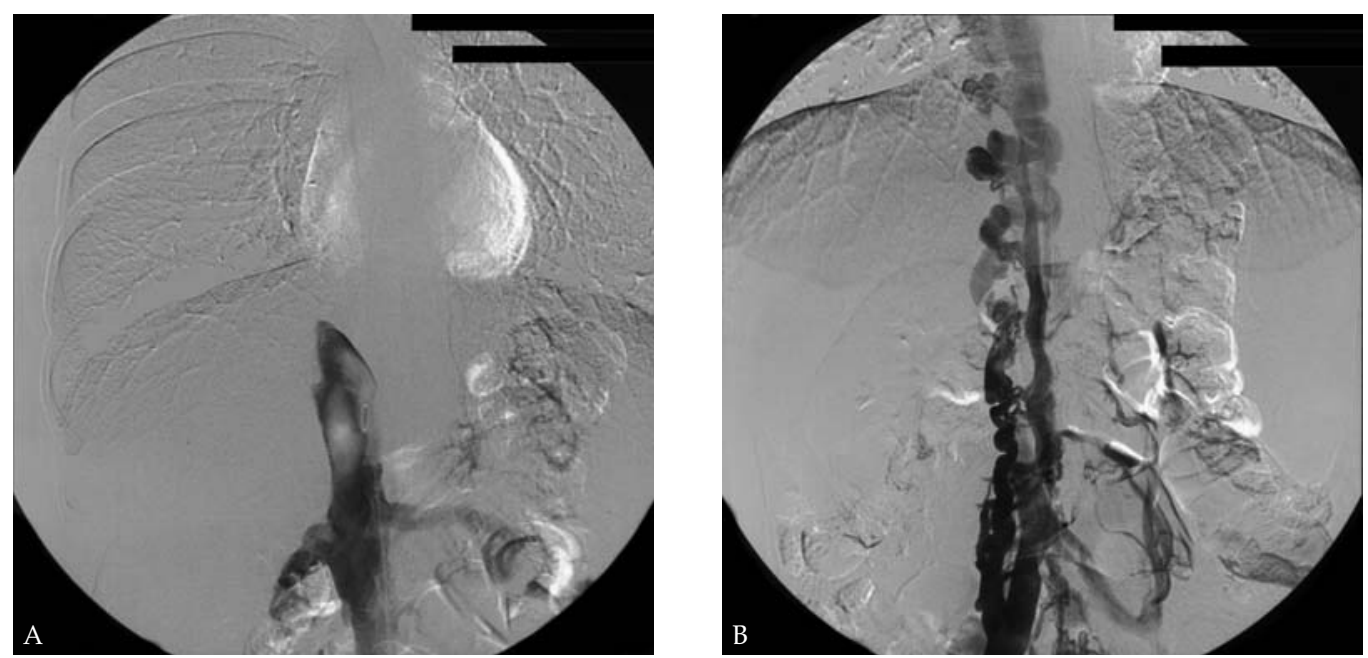

Figure 4. Cavography performed with $6 \mathrm{~F}$ pigtail catheter which could not passed into right atrium showed complete occlusion of distal IVC (A). Due to downward flow in the late series, markedly dilated and tortuous paraspinal collaterals were seen (B).

stent placement were considered but the patient did not accept the intervention.

\section{Discussion}

$\mathrm{OH}$ is a relatively new term proposed to define primary IVC thrombosis and membranous obstruction of the IVC. ${ }^{1}$ These two

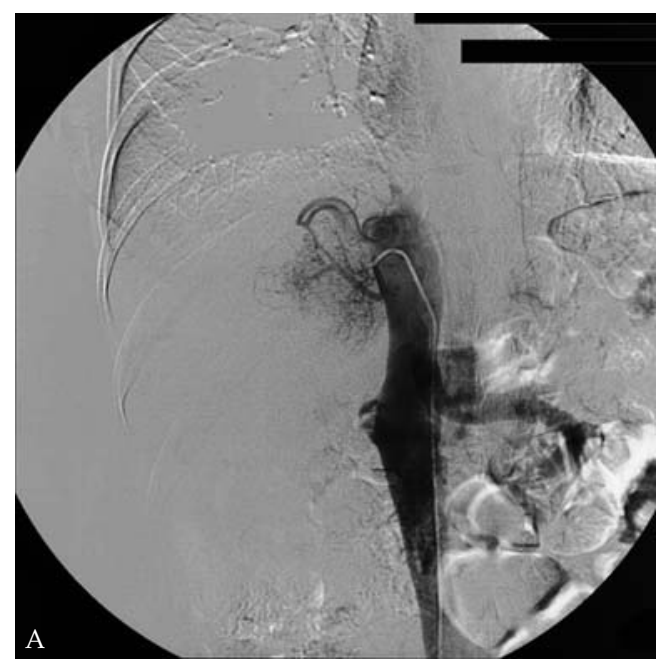

conditions were included in Budd-Chiari syndrome which was defined as hepatic venous outflow obstruction at any level from the small hepatic veins to the junction of the IVC and the right atrium, regardless of the cause of obstruction. ${ }^{2}$ Occlusion and stenosis of the IVC are squeals of primary IVC thrombosis. ${ }^{1}$ Although the majority of these cases are idiopathic, as in our case,

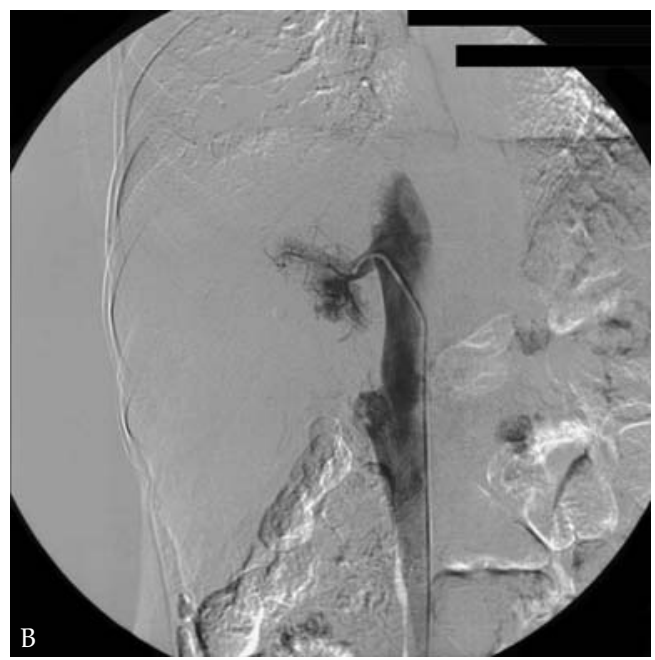

Figure 5. Selective hepatic vein injection showed a part consistent with hepatic vein confluence and tangle of vessels in the area of hepatic veins (A) and a small vein that had been seen during Doppler examination (B). 

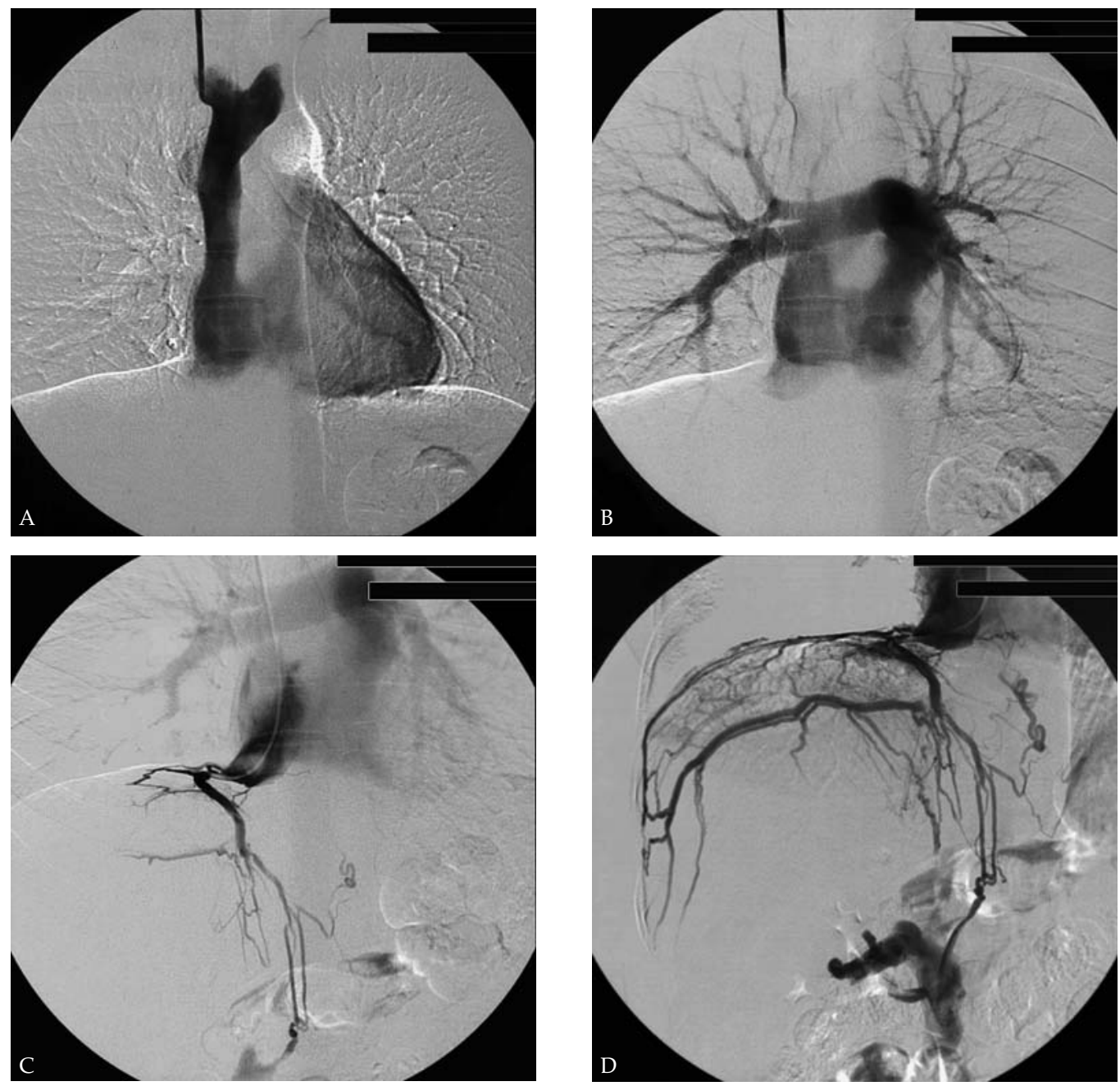

Figure 6. Superior venacavogram through introducer in right jugular vein shows no passage into IVC (A) and rapid filling of pulmonary arteries (B). Selective catheterization with a multipurpose catheter shows extrahepatic collaterals and filling of pulmonary arteries due to backflow (C), and in the later phases collateral connecting to occluded distal IVC appears and than IVC fills.

thrombotic or hypercoagulable conditions (like coagulation factor deficiency or mutation, myeloproliferative disorders, oral contraceptive use, pregnancy etc) could be observed in $\mathrm{OH}^{1}{ }^{1}$ If all hepatic veins become occluded, blood is coming from hepatic artery drain into either portal veins or collateral vessels. Retrograde flow in the portal vein occurs and portocaval shunts develop. As the occlusion progresses, blood circulation changes appear in and around liver. Intrahepatic or subcapsular hepatic venous collaterals are a distinctive feature of Budd-Chiari syndrome. ${ }^{2,3}$ In complete IVC obstruction, large shunts form between the IVC and the ascending lumbar vein continuous with the azygos and hemiazygos veins or paravertebral veins. The increased pressure in the ascending lumbar/hemiazygos and azygos veins may result in retrograde flow in the intercostal and subcutaneous veins. 
Due to mainly difficulties in the differential diagnosis of primary hepatic vein thrombosis and primary IVC thrombosis, the epidemiology of primary IVC thrombosis is not well established and adults are affected more frequently, but $\mathrm{OH}$ could be seen at any age..$^{1}$ The clinical presentation is acute and very serious like in the case of vena cava superior sindrom. ${ }^{1,4}$ Hepatomegaly, ascites, abdominal pain, fever and leg oedema appear quite fast, but the clinical course could be chronic with repeated acute episodes characterized by ascites, hepatomegaly, and pain. ${ }^{1}$ Due to the circulatory disturbances caused by IVC obstruction, patients commonly show marked venous dilatation over the body trunk. ${ }^{1}$ Our case had no visible venous dilatation over the body trunk possibly due to the collateral between occluded distal IVC and right atrium.

The radiological imaging provides a definitive diagnosis of $\mathrm{OH}$. In addition to demonstrating thrombus within the IVC and hepatic vein problems, ultrasonography also demonstrates caudate lobe enlargement, splenomegaly, ascites, stenotic veins, membrane, intrahepatic collateral veins connecting with enlarged hepatic veins, vein to-vein anastomoses, a patent and enlarged inferior right hepatic vein opening into the IVC, and absence of normal respiratory changes in the IVC. Doppler US could delineate flow disturbances and direction. CT and MRI assess hepatomegaly, ascites, enlarged caudate lobe, splenomegaly, collateral vessels, and the thrombus occluding the IVC. MRI depict the level of an obstructing membrane or web within the IVC. ${ }^{1}$ Hepatic venography delineates occluded veins, slow flow in attenuated venous branches, and rapid shunting into the portal vein. Additionally, wedged hepatic venography will demonstrate the "spider's web" network. ${ }^{1}$ Cavography is one of the most important imaging modality in the evaluation of IVC obstruction. It confirms the level of obstruction, occluding thrombus, mural thrombosis, membrane, and extrahepatic collateral vessels. ${ }^{1}$ Based on cavography findings three types of IVC obstruction were defined; type I, a thin membrane is present in the vena cava, type II, there is an absent segment of the IVC of variable length, type III, the vena cava is not visualized, and only dilated collateral channels are demonstrated. ${ }^{2}$ According to this classification, our case appears to be type II.

Although this disease classically treated by surgery (e.g. portal or mesenteric-systemic shunting, liver transplantation), interventional radiology procedures e.g. transjugular intrahepatic portosystemic shunting, percutaneous hepatic vein angioplasty and/ or stent placement also plays an important role in the management - like in the others severe vascular complicated cases. 3,5,6

\section{References}

1. Okuda K. Inferior vena cava thrombosis at its hepatic portion (obliterative hepatocavopathy). Semin Liver Dis 2002; 22: 15-26.

2. Sakamoto N, Koizumi K, Asahina Y, Tazawa J, Maeda M, Marumo F, et al. Primary Budd-Chiari syndrome due to complex venous anomalies. Abdom Imaging 1997; 22: 499-501.

3. Janssen HL, Garcia-Pagan JC, Elias E, Mentha G, Hadengue A, Valla DC; European Group for the Study of Vascular Disorders of the Liver. BuddChiari syndrome: a review by an expert panel. $J$ Hepatol 2003; 38: 364-71.

4. Vodváŕka P, Števerák P. Algorithm for percutaneous stenting in patients suffering from superior vena cava syndrome. Radiol Oncol 2000; 34: 349-55.

5. Wu T, Wang L, Xiao Q, Wang B, Li S, Li X, et al. Percutaneous balloon angioplasty of inferior vena cava in Budd-Chiari syndrome-R1. Int J Cardiol 2002; 83: 175-8.

6. Gjikolli B, Hadžihasanović B, Jaganjac S, Herceglija E, Nikšić M, Hadzimehmedagić A, et al. Treatment of complicated case with subclavia steal syndrome and stenosis of common iliac artery. Radiol Oncol 2008; 42: 1-12. 